\title{
Applicability of Recent Dyslipidemia Guidelines in Clinical Practice
}

\author{
Erwinanto \\ Department of Cardiology and Vascular Medicine, \\ Cardiovascular Division, Department of Internal Medicine, \\ Universitas Padjajaran - Dr. Hasan Sadikin General Hospital, Bandung, Indonesia
}

Corresponding author:

Erwinanto, MD, - email: ewnkar@yahoo.com

Department of Cardiology and Vascular Medicine, Cardiovascular Division, Department of Internal Medicine, Universitas Padjajaran

- Dr. Hasan Sadikin General Hospital

Jalan Pasteur 38, Bandung, Indonesia

\begin{abstract}
Atherosclerotic plaque rupture is closely related to acute coronary syndromes.Stabilization of atherosclerotic plaque which slashes plaque rupture is as importantas regression ofplaque size for reducing cardiovascular events. Dyslipidemia therapy targeting to decrease LDL cholesterol reduces cardiovascular events such as acute myocard infarct, stroke, and death which are suggested to be the result of plaque stabilization. Dyslipidemia therapy also regress atherosclerotic plaque into a smaller volume. Plaque regression improves coronary flow responsible for the reduction of myocardial infarction incidence in patients with coronary heart disease (CHD). This paper consists of two parts. The first part discusses the evidence of cardiovascular event reduction with statin. The second part describes dyslipidemia management based on the 2017 Indonesian Heart Association (PERKI) Guideline on the Management of Dyslipidemia
\end{abstract}

Dyslipidemia therapy stabilizes and regress atherosclerotic plaque

Dyslipidemia therapy has two purposes. The first is to stabilize atherosclerotic plaque from being ruptured, thus preventing acute coronary syndromes, stroke, and death. The second is to reduce plaque volume (plaque regression). Stable plaque consists of less macrophage and oxidized LDL and has thicker fibrous cap despite of normal plaque volume. Regressed plaque has smaller volume without changes of the plaque component and thickness of the fibrous cap.

The JUPITER study is a study with the objective to see the effect of lowering LDL cholesterol with rosuvastatin on cardiovascular events i.e. myocardial infarction and ischemia, stroke, and cardiovascular death. This study was conducted inhigh-risk subjects without cardiovascular disease (CVD). Therapy with rosuvastatin $20 \mathrm{mg}$ for 4 years has been shown to significantly reduce cardiovascular events by $44 \%$. The therapeutic effect is seen several months after starting treatmentthat indicates the stabilization of atherosclerotic plaques. The results of the JUPITER study support statin administration as soon as possible for patients with high cardiovascular risk.

Another study with rosuvastatin (ASTEROID study) showed that rosuvatatin $40 \mathrm{mg}$ for 2 years regressed atherosclerotic plaques in subjects with CHD. The results of the ASTEROID study provide support for 
administering high-dose statin therapy for CHD patients.

Management of dyslipidemia

Among the lipid profiles routinely examined, only LDL cholesterol is stronglyrelated to cardiovascular events in randomized clinical trials. This fact makesLDL cholesterol to be the primary target of dyslipidemia management. LDL cholesterol must be lowered to itstarget level before managing other lipids. Triglycerides reduce cardiovascular events in subjects with high cardiovascular riskonlyifLDL cholesterol has been lowered to target. There is no target for triglycerides. However, triglycerides should be lowered immediatelyif the concentration is $\geq 500 \mathrm{mg} / \mathrm{dl}$ to avoid acute pancreatitis. The secondary target of treatment is nonHDL cholesterol calculated by reducing the concentration of HDL to total cholesterol.

Total and HDL cholesterol although routinely examined are not the target of treatment given the lack of adequate clinical evidence. Total cholesterol and HDL cholesterol are used in cardiovascular risk stratification. Routine examination forother lipid profiles is not advisedexcept in certain clinical condition.

The management discussed here refers to the 2017 PERKI Dyslipidemia Management Guideline. The guidelines introduce step-by-step approach (5 steps) that is intended to help physician to deal with lipid management.

The first step

Screening for dyslipidemia is always indicated in subjects withclinical manifestations of CVD, in clinical conditions associatedwith increased risk for CVD and whenever risk factor screeningis considered. Recognition of dyslipidemia requires information of CVD risk level i.e. very high, high, moderate, and low. The cut- off points used to definerisk level are partly arbitrary and based on the risk level at which treatment benefit is evident in clinical trials.

Risk level is influenced by the presence of 5 clinical conditions that needs to be investigated,i.e.atherosclerotic cardiovascular diseases, diabetes mellitus (DM), chronic kidney disease (CKD), uncontrolled hypertension, and familial hypercholesterolemia. Atherosclerotic cardiovascular diseaserefers to atherosclerosis ofany blood vessel such as coronary (CHD), brain (ischemic stroke), and peripheral vessels (peripheral arterial disease). Chronic kidney disease (CKD) is defined asGFR of less than 60 $\mathrm{ml} /$ minute/1.73 $\mathrm{m}^{2}$.

Patients with atherosclerotic cardiovascular diseases, CKD with a GFR of less than $30 \mathrm{ml} /$ minute $/ 1.73 \mathrm{~m}^{2}$, or DM with albuminuria or other risk factors are classified as very high risk. The high cardiovascular risk is proposed for those with DM who do not meet the very high risk criteria, CKD with a GFR of 30-59 $\mathrm{ml} /$ minute/1.73 $\mathrm{m}^{2}$, severe hypertension (systolic BP exceeds $160 \mathrm{~mm} \mathrm{Hg}$ ) uncontrolled with drugs, and familial hypercholesterolemia (total cholesterol above $310 \mathrm{mg} / \mathrm{dl}$ ). For all other patients, CVD risk is estimated using the SCORE chart.See the 2017 PERKI Dyslipidemia Management Guideline for detail of SCORE estimation system. The guideline can be downloaded for free at www.inaheart.com.

The second step

The second step is defining LDL cholesterol treatment target based on patient's risk level. A person with LDL cholesterol above the treatment target is defined as dyslipidemia,in whom LDL cholesterol lowering treatment should be initiated. The LDL cholesterol treatment 
target for those with very high, high, or middle/low risk is $<70 \mathrm{mg} / \mathrm{dl},<100 \mathrm{mg} / \mathrm{dl}$, or $<115 \mathrm{mg} / \mathrm{dl}$ respectively.

The third step

The third step is to develop a strategy to achieve the target of LDL cholesterol. The strategy requires information of a) LDL cholesterol concentration at baseline, b) LDL cholesterol target, and c) the available treatment choices. The strategy should always include therapeutic lifestyle changes (not discussed in this paper) with or without LDL cholesterol-lowering drug. Drug therapy is indicated for all patients with LDL cholesterol above treatment target, except for patients at low risk and myocardial infarction. Drug therapy for low-risk patients is indicated if the baseline LDL cholesterol is $\geq 190 \mathrm{mg} / \mathrm{dl}$. Patients with acute myocardial infarction should receive LDL cholesterol-lowering therapy regardless of their baseline LDL cholesterol.

Given that proprotein convertase substilysin kexin9 (PCSK9) inhibitor is not available in Indonesia, discussion of LDL cholesterol lowering drug treatment refers to statin and its combination with ezetimibe. Statin/ezetimibe combinationis indicated for those with high and very high cardiovascular risk if the baseline LDL cholesterol is $>200 \mathrm{mg} / \mathrm{dl}$ and $>135 \mathrm{mg} / \mathrm{dl}$ respectively. High-dose statins cannot reduce such high LDL cholesterol to target. The combination of statin/ezetimibe is also indicated for those in whom LDL cholesteroltarget is not reached despite treatment with high-dose statins.

The fourth step
The fourth step is the management of non-HDL cholesterol for patients with high and very high cardiovascular risk in whom LDL cholesterol has reached the target level and the non-HDL cholesterol is still $>30 \mathrm{mg} / \mathrm{dl}$ above the target of LDL cholesterol. Thus, non-HDL cholesterol therapy is initiated in high and very high risk patients if $>130 \mathrm{mg} / \mathrm{dl}$ and $>100 \mathrm{mg} / \mathrm{dl}$ respectively. Use fibrate to lower non-HDL cholesterol. Fenofibrates is preferred over gemfibrozil.

The fifth step

The final step is monitoring the LDL cholesterol, SGPT, and creatinine kinase. Before starting statin therapy, check the LDL cholesterol twice except for very high risk patients or those with acute coronary syndrome.Repeat the test after 1-12 weeks. Start drug treatment immediately for patients at very-high risk or with acute coronary syndrome. Repeat LDL cholesterol test 4-12 weeks after treatment initiation or dose change. If the LDL cholesterol concentration has reached the treatment target, repeat the test yearly.

SGPT is examined before statin therapy and 8-12 weeks thereafter or after changing the dose. If SGOT increases $<3 x$ upper limit of normal (ULN), continue statin and repeat SGPT testing after 4-6 weeks. Postpone or stop statin therapy for 4-6 weeks if SGPT is $\geq 3 x$ ULN and repeat the test before resuming statin. Therapy can be resumed if SGPT has returned to normal. Creatinine kinase is examined if there is unexplained muscle pain (myalgia) that does not respond to pain killer. Follow the algorithm below if there is an increase in creatinine kinase. 


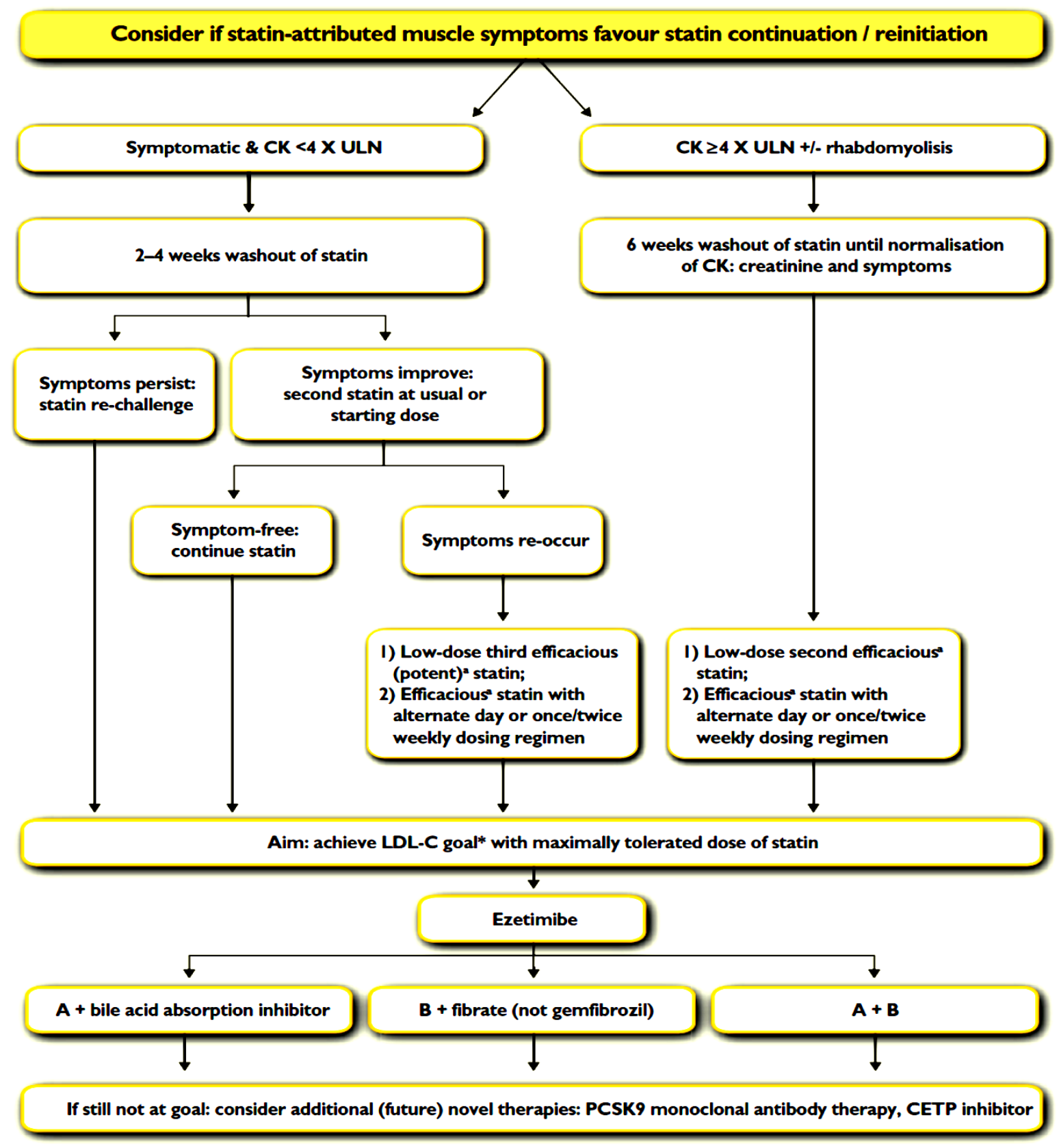

CETP = cholesteryl ester transfer protein; $C K=$ creatine kinase; $L D L-C=$ low-density lipoprotein cholesterol; PCKS9 = propoteinonvertasesubtilisin/kesin type 9; ULN = upper limit of the normal range. aEfficacious statin such as atorvastatin or rosuvastatin

(Catapano AL, et al. Eur Heart J 2016. doi:10.1093/eurheartj/ehw272) 